\title{
Erratum
}

Journal of Inclusion Phenomena and Macrocyclic Chemistry (2005) 52: 51-54

(C) Springer 2005 DOI $10.1007 / \mathrm{s} 10847-004-3842-\mathrm{x}$

\section{The Effect of Benzo Substitution on Complexation of Diaza 18-Crown-6 Ethers Derivatives with $\mathrm{NaClO}_{4}$}

\section{M.ZAFER KÖYLÜ ${ }^{2}$, NADIR DEMIREL ${ }^{1, *}$, F.DOGANEL POLAT ${ }^{3}$, ALI YILMAZ ${ }^{2}$, HALIL HOSGÖREN ${ }^{1}$ and METIN BALCI ${ }^{3}$}

${ }^{1}$ Department of Chemistry, Faculty of Science, METU, 06100, Ankara, Turkey; ${ }^{2}$ Department of Chemistry, Faculty of Science, University of Dicle, 21280, Diyarbakır, Turkey; ${ }^{3}$ Department of Physics, Faculty of Science, University of Dicle, 21280, Diyarbakir, Turkey

A part of Table 1 was missing from this article. Please find the complete table below.

Table 1. ${ }^{1} \mathrm{H}$ chemical shifts of macrocyclic protons $(\mathrm{H}-1-\mathrm{H}-6)$ at $\mathrm{NaClO}_{4}$ concentrations (in $\mathrm{CD}_{3} \mathrm{CN}$ )

\begin{tabular}{|c|c|c|c|c|c|c|}
\hline & H-1 & H-2 & H-3 & H-4 & H-5 & H-6 \\
\hline \multicolumn{7}{|l|}{ Free Crown 1} \\
\hline$\delta(\mathrm{ppm})$ & 3.99 & 3.20 & 2.53 & - & - & - \\
\hline$\Delta \delta(\mathrm{ppm})[\mathrm{NaClO} 4] /[$ Crown] $: 1: 1$ & 0.16 & -0.23 & 0.05 & - & - & - \\
\hline \multicolumn{7}{|l|}{ Free Crown 2} \\
\hline$\delta(\mathrm{ppm})$ & 4.00 & 3.23 & 3.73 & - & - & - \\
\hline$\Delta \delta(\mathrm{ppm})[\mathrm{NaClO} 4] /[$ Crown $]: 1: 1$ & 0.19 & -0.25 & -0.01 & - & - & - \\
\hline \multicolumn{7}{|l|}{ Free Crown 3} \\
\hline$\delta(\mathrm{ppm})$ & 4.13 & 3.01 & 2.47 & 2.86 & 3.55 & 3.51 \\
\hline$\Delta \delta(\mathrm{ppm})[\mathrm{NaClO} 4] /[$ Crown] $: 1: 1$ & 0.05 & 0.21 & 0.03 & -0.21 & -0.02 & 0.06 \\
\hline
\end{tabular}

* Author for Correspondence. E-mail: demireln@dicle.edu.tr 